\title{
Reduced Ischemia in the Medial Vestibular Nucleus after Premedication of Cilostazol and Ginkgo Biloba Extract in Global Hypoperfusion Rat Model
}

\section{Lee $\mathrm{JO}^{1}$, Kim $\mathrm{HJ}^{2}$ and $\mathrm{Kim} \mathrm{JS}^{\star 3}$}

${ }^{1}$ Biomedical Research Institute, Seoul National University Bundang Hospital, Korea

${ }^{2}$ Department of Biomedical Laboratory Science, Kyungdong University, Goseong-gun, Gangwon-do, Korea

${ }^{3}$ Department of Neurology, Seoul National University College of Medicine, Seoul National University Bundang Hospital, Seongnam, Korea

${ }^{*}$ Corresponding author: Kim JS, Department of Neurology, Seoul National University College of Medicine, Seoul National University Bundang Hospital, Seongnam, Korea, Fax: +82-31-719-6828, Tel: +82-31-7877463, E-mail: jisookim@snu.ac.kr

Citation: Lee JO, Kim HJ, Kim JS (2015) Reduced Ischemia in the Vestibular Nucleus after Premedication of Cilostazol and Ginkgo Biloba Extract in Global Hypoperfusion Rat Model. J Neurol Neurol Disord 2(2): 203. doi: $10.15744 / 2454-4981.2 .203$

Received Date: March 26, 2015 Accepted Date: June 25, 2015 Published Date: June 26, 2015

\begin{abstract}
Objective: The aim of this study was to determine preventive effects of premedication with combination of cilostazol and ginkgo biloba extract during vertebrobasilar ischemia (VBI) induced by global hypoperfusion in rats.

Methods: We randomly allocated Sprague-Dawley male rats weighting 240 250 g to the premedication or control group. The premedication group received a combination of cilostazol $(25 \mathrm{mg} / \mathrm{kg} /$ day $)$ and ginkgo biloba extract $(20 \mathrm{mg} / \mathrm{kg} / \mathrm{day})$ per os for 7 days before the induction of transient global ischemia with 4-vessel-occlusion (4VO) for 20 minutes. Both vertebral arteries (VAs) were permanently occluded using an electrocauterization and bilateral common carotid arteries (CCAs) were temporarily occluded for 20 minutes with a ligation. One hour after reperfusion of the CCAs, the animals were euthanized and subjected to c-Fos immunohistochemical staining of the medial vestibular nucleus (MVN), which has been found to be the infratentorial region most sensitive to ischemia.

Results: The rats in the premedication group showed a significant decrease in the number of neuron showing c-Fos protein expression in the MVN compared to the control group ( $93.5 \pm 18$ vs. $64.2 \pm 15$, $t$-test, $p=0.002$ ).

Discussion: Combination of cilostazol and ginkgo biloba extract appears to have a protective effect on the vestibular system during transient global ischemia, and may be considered in patients with recurrent vertigo from VBI.
\end{abstract}

Keywords: Renexin ${ }^{\circledR}$ Ischemia; Vertigo; Medial vestibular nucleus; Rat

\section{Introduction}

Approximately $20 \%$ of cerebral ischemia is known to develop in the territory of the vertebrobasilar artery and the most common symptoms of vertebrobasilar ischemia (VBI) are dizziness and vertigo [1]. Isolated vertigo without other neurologic symptoms has generally been ascribed to peripheral vestibular diseases [1]. Of the patients with vertigo due to VBI, however, $62 \%$ had at least one isolated episode of vertigo, and 19\% developed vertigo as the initial symptom [2]. A recent study also found that the patients hospitalized with isolated vertigo have a threefold higher risk for strokes than the general population during the 4-year follow-up [3].

The lesion sites responsible for isolated vascular vertigo remain to be elucidated. Isolated vertigo observed in VBI may be ascribed to selective involvement of the terminal branch supplying the vestibular end organs. Compression of the vertebral artery terminating in the posterior inferior cerebellar artery (PICA) alone can induce vertigo, transient ischemia of the inferior cerebellum or lateral medulla may be a mechanism of isolated vertigo in human [1]. Indeed, using a transient global hypoperfusion rat model, of all the structures in the brainstem and cerebellum, the medial vestibular nucleus (MVN) is most vulnerable to ischemia [4].

In suspected patients with isolated episodes of vertigo from VBI, it is important to initiate appropriate treatments for prevention of further attacks and completed stroke, and antiplatelet agents may be an option. Renexin ${ }^{\circledast}$ (SK Chemical, Korea), a mixed composition of cilostazol and ginkgo biloba extract $\left(\mathrm{GB}_{\text {ext }}\right)$, was recently introduced into clinical practice. Renexin ${ }^{\otimes}$ has been found to have more neuroprotective effects than cilostazol or $\mathrm{GB}_{\text {ext }}$ alone in animal studies [5]. However, no study has attempted prevention effects of Renexin ${ }^{\circledast}$ on vertigo due to ischemia. In this study, we aimed to determine neuro-preventive effects of Renexin ${ }^{\circledR}$ premedication on the MVN during ischemia induced by global hypoperfusion in rats. 


\section{Materials and Methods}

\section{Animals}

A pair of Sprague-Dawley male rats weighing 240 250 g was randomly assigned to the control and premedication group for each experiment. The rat in the premedication group had taken Renexin ${ }^{\oplus}$ for 7 days and the one in the control group was kept without medication in the same conditions before the induction of transient global hypoperfusion. The experiment was repeated until five pairs of rats completed the whole procedures. Treatment of animals was in accordance with the guidelines regarding the care and use of animals for experimental procedures, and was approved by the Institutional Animal Care and Use Committee of Seoul National University Bundang Hospital (IACUC No. BA1108-088-049-01). All efforts were made to minimize the number of animals used and their sufferings.

\section{Premedication}

Renexin $^{\oplus}$ containing cilostazol (25 mg/kg/day) and $\mathrm{GB}_{\text {ext }}(20 \mathrm{mg} / \mathrm{kg} /$ day $)$ was supplied in a powder form by SK Chemical Co. (Korea). Just before administration, the powder ( $45 \mathrm{mg} / \mathrm{kg} /$ day) was suspended with $0.5 \%$ carbosyl methyl cellulose (Sigma), and was introduced via oral gavage in a volume of $10 \mathrm{ml} / \mathrm{kg}$.

\section{Experimental procedures}

We induced posterior circulation ischemia by modifying the 4-vessel-occlusion (4VO) technique described in detail previously [6]. Briefly, all animals were anesthetized with an intraperitoneal injection of Zoletil (Tiletamine/Zolazepam, $30 \mathrm{mg} / \mathrm{kg}, \mathrm{VIRBAC}$, France) and Rompun (Xylazine, $10 \mathrm{mg} / \mathrm{kg}$, Bayer, Korea). The first incision was made behind the occipital bone directly overlying the first two cervical vertebrae. Both vertebral arteries were then electrocauterized and permanently occluded using an electrocautery needle. Then, with the rats in the supine position, each common carotid artery (CCA) was isolated from the vagus nerve and 3.0 silk surgical suture loosely placed around it, and then both sutures were tightened simultaneously. Subsequently, the incision was closed with a single suture.

After occlusion for 20 minutes [7], the sutures were removed from the CCAs and restoration of the carotid blood flow was confirmed with direct observation. During the procedures, body temperature was maintained $37.5 \pm 0.5^{\circ} \mathrm{C}$ with a heat lamp.

\section{Histopathologic studies}

One hour after reperfusion of both CCAs, the animals were euthanized and subjected to immunohistochemical studies. Briefly, the animals were deeply anesthetized with intraperitoneal injection of Zoletil and Rompun, and sequentially perfused through the ascending aorta with $300 \mathrm{ml}$ of normal saline, and $300 \mathrm{ml}$ of $4 \%$ formaldehyde. Then, the animals were euthanized, and the perfused brain was removed for overnight fixation in the same fixative. The posterior part of the brain, including the vestibular nuclei, was embedded in paraffin. Subsequently, $20 \mu \mathrm{m}$-thick sections were obtained from -11 to $-12 \mathrm{~mm}$ relative to the bregma.

\section{Immunohistochemistry for c-Fos}

To retrieve antigen for c-Fos immunohistochemistry, the brain sections were heated for 25 minutes in $10 \mathrm{mM}$ citrate buffer within a microwave. Then the sections were incubated for 15 minutes with $3 \%$ hydrogen peroxide $\left(\mathrm{H}_{2} \mathrm{O}_{2}\right)$ and blocked with $3 \%$ normal goat serum for one hour. Subsequently the sections were incubated overnight at $4{ }^{\circ} \mathrm{C}$ with c-Fos polyclonal antibody (1:1000, Santa Cruz Biotechnology, Inc., USA). At the following day, the sections were incubated with a biotinylated secondary antibody (goat anti-rabbit, 1:500, DAKO, Denmark). These were followed by an avidin-biotin peroxidase method (ABC Elite Kit, Vector Laboratories, USA) and processed with 3,3'-diaminobenzidine (DAB, DAKO, Denmark), which resulted in a brown reaction product. After the DAB reaction, the sections were dehydrated and cover-slipped with Permount (Fisher Chemical, USA). The supraoptic nucleus region inducing c-Fos positive neurons by injection of hypertonic saline served as a positive staining control.

\section{Quantitative analysis}

After consecutive three sections were selected at a $40 \mu \mathrm{m}$ interval through the whole level of the MVN (bregma $-11.60 \sim-11.80$ $\mathrm{mm}$ ), those were examined and images were acquired with a Zeiss Axioskop 40 microscope (Carl Zeiss Micorscopy, Germany) to localize and count c-Fos positive neurons. Based on the previously developed atlas [8], the brown c-Fos positive neurons were automatically captured and computerized counting was performed with a software using the Auto Measure Program Wizard (Axio Vision version 4.8) adopted in Zeiss Axio observer microscope (Carl Zeiss Micorscopy, Germany). The c-Fos positive cells on either side of the sections were counted independently, so that any asymmetry in the sectioning procedure would not affect the accuracy of the cell counting. After uniform adjustment of the brightness and contrast of each image file, at least double counts per each nucleus were performed for each slide, and the average number of c-Fos positive neurons was calculated.

\section{Statistical analyses}

The sum of c-Fos positive neurons on either side of the sections was compared between the control and Renexin groups. All the quantitative data are presented as the mean \pm SD. Statistical analysis was performed using $t$-test, and $p<0.05$ were considered statistically significant. 


\section{Results}

The rats in either group well tolerated the surgical procedures without significant bleedings. Only a rat in the control group died one hour after reperfusion. The control group (4VO for 20 minutes) showed a markedly increased expression of c-Fos protein in the MVN. Also, the premedication group showed an increase of c-Fos positive neurons in the MVN after 20 minutes of $4 \mathrm{VO}$ (Figure 1). However, the number of c-Fos positive neurons was significantly lesser in the premedication group than in the control group ( $93.5 \pm 18$ vs. $64.2 \pm 15$, $t$-test, $p=0.002$, Figures 1 and 2 ).

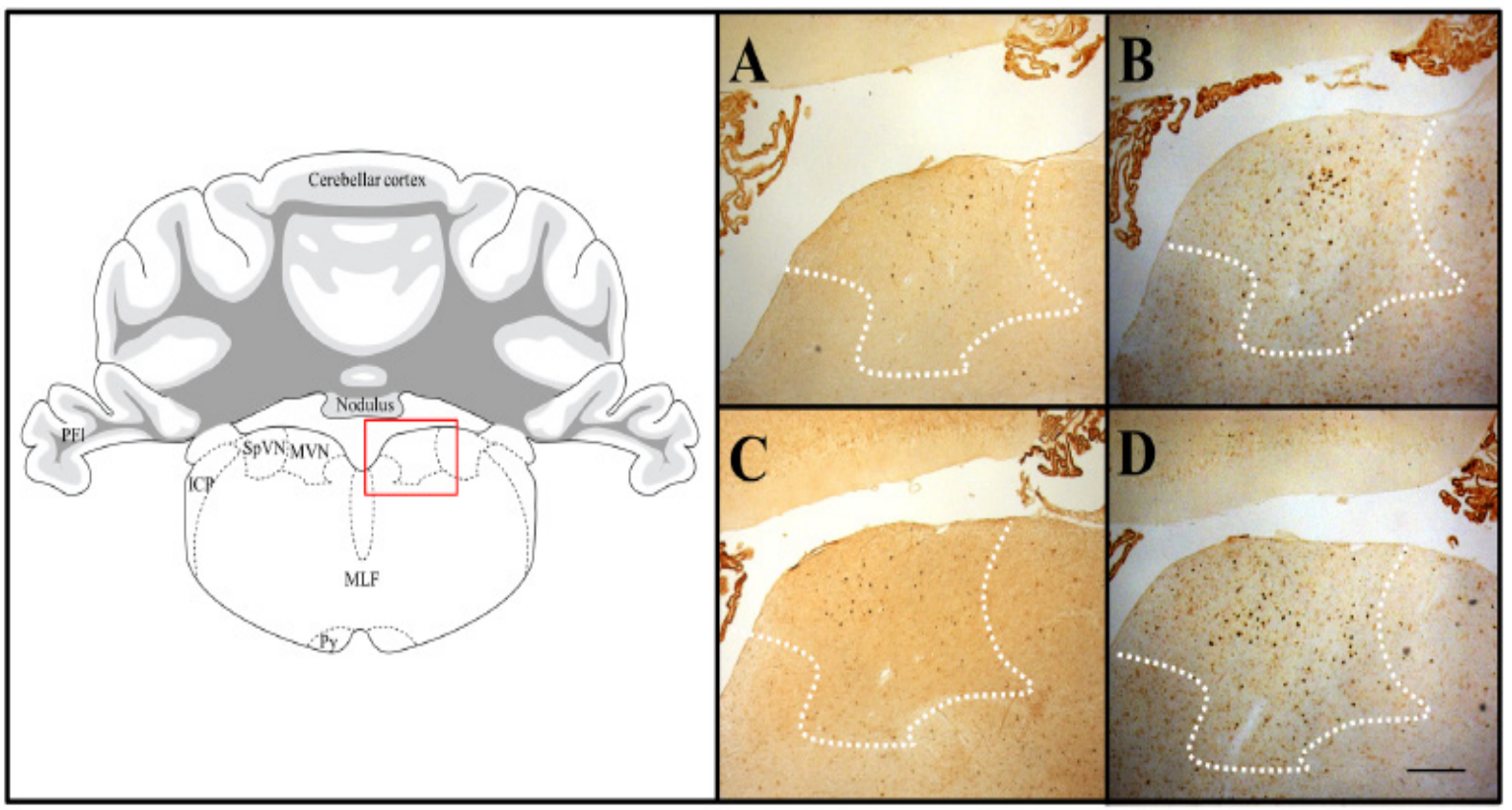

Figure 1: After 4-vessel occlusion (VO) for 20 minutes, the medial vestibular nucleus (MVN) shows decreased expression of c-Fos in the premedication group $(\mathrm{A}, \mathrm{C})$ compared with the control group $(\mathrm{B}, \mathrm{D})$. A \& B are at $-11.64 \mathrm{~mm}$ and $\mathrm{C} \& \mathrm{D}$ are at $-11.76 \mathrm{~mm}$ from the bregma. The area demarcated by the white dotted line corresponds to the MVN. The premedication group had taken a combination of cilostazol ( $25 \mathrm{mg} / \mathrm{kg} /$ day) and ginkgo biloba extract $(20 \mathrm{mg} / \mathrm{kg} /$ day $)$ per os for 7 days before induction of transient global ischemia using $4 \mathrm{VO}$ for 20 minutes. The scale bar is $200 \mu \mathrm{m}$. ICP, inferior cerebellar peduncle; MLF, medial longitudinal fasciculus; PFl, paraflocculus; py, pyramidal tract; $\mathrm{SpVN}$, spinal vestibular nucleus

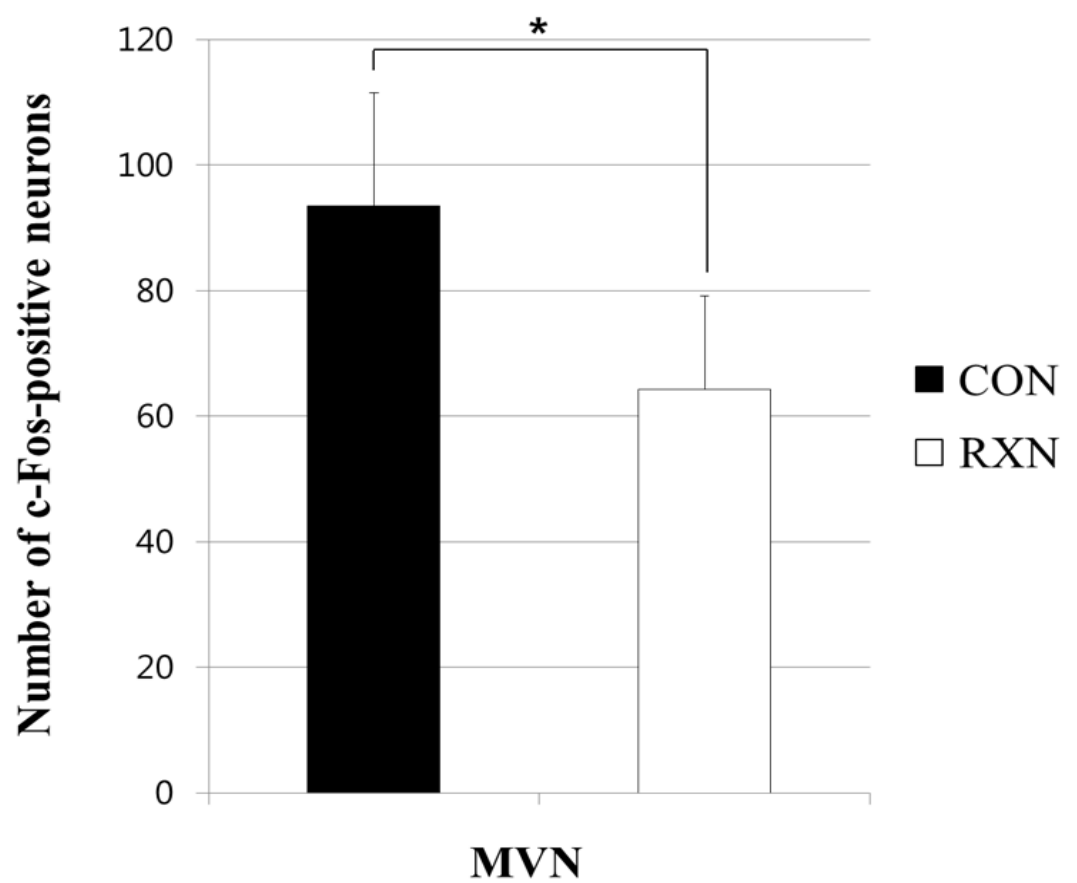

Figure 2: The number of c-Fos positive neurons in the MVN was significantly lesser in the premedication group than in the control group ( $93.5 \pm 18$ vs. $64.2 \pm 15$, $t$-test, $p=0.002$ ) after 4 -vessel occlusion for 20 minutes. CON, the control group; RXN, the premedication group; MVN, the medial vestibular nucleus 


\section{Discussion}

Brain ischemia leads to a cessation of ATP generation in the affected areas, and then causes generalized depolarization, release of excitatory transmitters in the selectively vulnerable neurons, and opening of the voltage-dependent and glutamate-regulated calcium channels [9]. The resultant $\mathrm{Ca}^{2+}$ influx itself stimulates glutamate release, which consistently activates the N-methyl-Daspartate (NMDA) receptor-operated calcium channels and causes excessive cytosolic $\mathrm{Ca}^{2+}$. At this stage, the immediate early

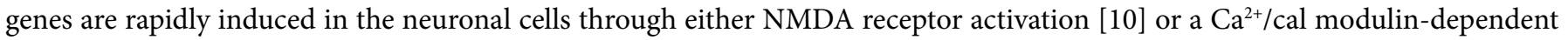
protein kinase-activated phosphorylation cascade [11].

The immediate early genesc-fos, jun and zif 168 are a member of proto-oncogene families, and is a well-known marker of neuronal activity by virtue of its very rapid expression in the neurons in response to environmental stimuli [12]. In response to cerebral ischemia, neuronal c-fos mRNA expression is increased in the central nervous system [13]. The results of numerous in vivo and in vitro studies have shown that induction of $c$-Fos protein precedes neuronal death and participates in neuronal injury after ischemia [14]. Likewise Renexin ${ }^{\oplus}$, the dextromethorphan, a neuroprotective drug, reduced an expression of c-Fos protein one hour after ischemia, and also protected delayed neuronal degeneration in the CA 1 region of the hippocampus $[15,16]$. These results suggest that reduction of c-Fos expression one hour after ischemia may be positively correlated with survival of neurons.

The MVN receives peripheral vestibular inputs and participates in the control of posture and movements. The MVN also influences sympathetic outflow and blood pressure $[17,18]$. Abnormal stimulation of the MVN may evoke nausea, vomiting, vertigo. The MVN normally maintains a balance of action potentials by an inhibitory input and two sources of excitatory tone [19]. Excitation of the vestibular neurons and resultant increase in intracellular $\mathrm{Ca}^{2+}$ concentration induce c-Fos protein expression in the MVN. In our previous study to determine ischemic vulnerability of the structures in the brainstem and cerebellum in rats using the same global hypoperfusion model, c-Fos expression was first detected in the MVN after 4VO for 20 minutes, which indicates that the MVN is most vulnerable to ischemia of all the structures in the brainstem and cerebellum.

Cilostazol, 6-[4-(1-cyclohexyl-1H-tetrazol-5-yl) butoxy]-3,4-dihydro-2-[1H]-quinolinone, is known as a potent reversible phosphodiesterase Ш (PDE3) inhibitor. Inhibition of phosphodiesterase allows for increased availability of cyclic adenosine monophosphate (CAMP) that mediates inhibition of platelet aggregation, vasodilation and vascular antiproliferative response [20]. Cilostazol, which effectively scavenges hydroxyl and peroxyl, was also found to possess neuroprotective and antiapoptotic properties in focal cerebral ischemic models [21].

$\mathrm{GB}_{\text {ext }}$ is one of the most popular herbal products, consisting of flavonoid glycosides (24\%), terpene lactones (6\%, including gingkolides A, B, C, J and bilobalide) and other substances (70\%, such as proanthocyanidins, organic acids, and sugars, etc.) [22]. $\mathrm{GB}_{\text {ext }}$ is known to have extensive protective effects on central nervous and circulation systems. By increasing blood flow, $\mathrm{GB}_{\text {ext }}$ improves peripheral arterial insufficiency, and reduces blood viscosity and thrombocyte aggregation induced by ischemia [20]. $\mathrm{GB}_{\text {ext }}$ also influences the neurotransmitters. $\mathrm{GB}_{\text {ext }}$ increases the level of $\gamma$-aminobutyric acid (GABA) as well as the activity

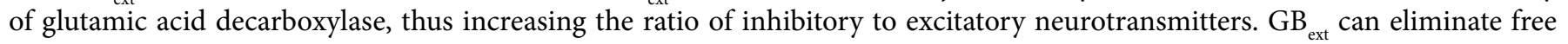
oxygen radicals, reduce lipid peroxidation, and facilitate the systhesis and release of prostacyclin [22].

In a previous study, a combination cilostazol and $\mathrm{GB}_{\text {ext }}$ exerted an enhanced therapeutic effect. It protected against auditory and vestibular damaged induced by CDDP (cis-diamm-ine-dichloproplatinum), probably by virtue of inhibiting reactive oxygen species (ROS) production, and mitochondrial and extracellular regulated kinase (ERK) pathways [23]. Also, it reduced cognitive decline caused by chronic hypoperfusion in the rat 2VO model [19]. Furthermore, it could reduce the serious side effects of antithrombotic agents, such as increased bleeding time, prolongation of prothrombin time (PT) and activated partial

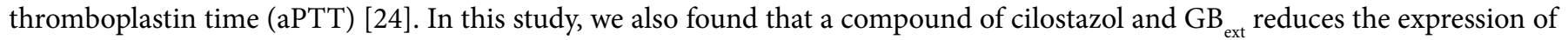
c-Fos protein that is a marker of cell apoptosis induced by ischemia.

In conclusion, we demonstrated that pre-treatment with combination of cilostazol and $\mathrm{GB}_{\text {ext }}$ has a neuroprotective effect on the MVN that is known to be most vulnerable to ischemia during global hypoperfusion in rats. Combination of cilostazol and $\mathrm{GB}_{\text {ext }}$ may be considered in patients presenting with recurrent vertigo from VBI.

\section{Acknowledgement}

The study was supported by a grant (No.06-2013-111) from SK chemical Co., Ltd.

\section{References}

1. Kim JS, Lee H (2013) Vertigo due to posterior circulation stroke. Semin Neurol 33: 179-84.

2. Grad A, Baloh RW (1989) Vertigo of vascular origin. Clinical and electronystagmographic features in 84 cases. Arch Neurol 46: $281-4$.

3. Lee CC, Su YC, Ho HC, Hung SK, Lee MS, et al. (2011) Risk of stroke in patients hospitalized for isolated vertigo: a four-year follow-up study. Stroke 42: 48-52.

4. Lee JO, Park SH, Kim HJ, Kim MS, Park BR, et al. (2014) Vulnerability of the vestibular organs to transient ischemia: Implications for isolated vascular vertigo. Neurosci Lett 558: 180-5.

5. Kwak PA, Lim SC, Han SR, Shon YM, Kim YI (2012) Supra-additive neuroprotection by renexin, a mixed compound of ginkgo biloba extract and cilostazol, against apoptotic white matter changes in rat after chronic cerebral hypoperfusion. J Clin Neurol 8: 284-92. 
6. Pulsinelli WA, Brierley JB (1979) A new model of bilateral hemispheric ischemia in the unanesthetized rat. Stroke 10: 267-72.

7. Macovschi O, Prigent AF, Nemoz G, Pacheco H (1987) Effects of an extract of Ginkgo biloba on the 3',5'-cyclic AMP phosphodiesterase activity of the brain of normal and triethyltin-intoxicated rats. J Neurochem 49: 107-14.

8. Paxinos G, Watson C (2007) The rat brain in stereotaxic coordinates, Academic Press, Elsevier, Amsterdam; Boston.

9. Siesjo BK (1992) Pathophysiology and treatment of focal cerebral ischemia. Part II: Mechanisms of damage and treatment. J Neurosurg 77: 337-54.

10. Cole AJ, Saffen DW, Baraban JM, Worley PF (1989) Rapid increase of an immediate early gene messenger RNA in hippocampal neurons by synaptic NMDA receptor activation. Nature 340: 474-6.

11. Bading H, Ginty DD, Greenberg ME (1993) Regulation of gene expression in hippocampal neurons by distinct calcium signaling pathways. Science 260: $181-6$.

12. Morgan JI, Curran T (1991) Stimulus-transcription coupling in the nervous system: involvement of the inducible proto-oncogenes fos and jun. Annu Rev Neurosci 14: 421-51.

13. Kogure K, Kato H (1993) Altered gene expression in cerebral ischemia. Stroke 24: 2121-7.

14. Preston GA, Lyon TT, Yin Y, Lang JE, Solomon G, et al. (1996) Induction of apoptosis by c-Fos protein. Mol Cell Biol 16: 211-8.

15. Bokesch PM, Marchand JE, Connelly CS, Wurm WH, Kream RM (1994) Dextromethorphan inhibits ischemia-induced c-fos expression and delayed neuronal death in hippocampal neurons. Anesthesiology 81: 470-7.

16. Nyitrai G, Puskás L, Antal K, Takács V, Sass M, et al. (2005) Preconditioning-specific reduction of c-fos expression in hippocampal granule and pyramidal but not other forebrain neurons of ischemic brain: a quantitative immunohistochemical study. Neurosci Lett 381: 344-9.

17. Jáuregui-Renaud K, Hermosillo AG, Gómez A, Márquez MF, Cárdenas M, et al. (2003) Vestibular function interferes in cardiovascular reflexes [corrected]. Arch Med Res 34: 200-4.

18. Yates BJ (1996) Vestibular influences on the autonomic nervous system. Ann N Y Acad Sci 781: 458-73.

19. Gallagher JP, Phelan KD, Shinnick-Gallagher P (1992) Modulation of excitatory transmission at the rat medial vestibular nucleus synapse. Ann N Y Acad Sci 656: 630-44.

20. Haslam RJ, Dickinson NT, Jang EK (1999) Cyclic nucleotides and phosphodiesterases in platelets. Thromb Haemost 82: $412-23$.

21. Choi JM, Shin HK, Kim KY, Lee JH, Hong KW (2002) Neuroprotective effect of cilostazol against focal cerebral ischemia via antiapoptotic action in rats. J Pharmacol Exp Ther 300: 787-93.

22. Smith JV, Luo Y (2004) Studies on molecular mechanisms of Ginkgo biloba extract. Appl Microbiol Biotechnol 64: 465-72.

23. Tian CJ, Kim YJ, Kim SW, Lim HJ, Kim YS, et al. (2013) A combination of cilostazol and Ginkgo biloba extract protects against cisplatin-induced Cochleovestibular dysfunction by inhibiting the mitochondrial apoptotic and ERK pathways. Cell Death Dis 4: e509.

24. Ryu KH, Han HY, Lee SY, Jeon SD, Im GJ, et al. (2009) Ginkgo biloba extract enhances antiplatelet and antithrombotic effects of cilostazol without prolongation of bleeding time. Thromb Res 124: 328-34.

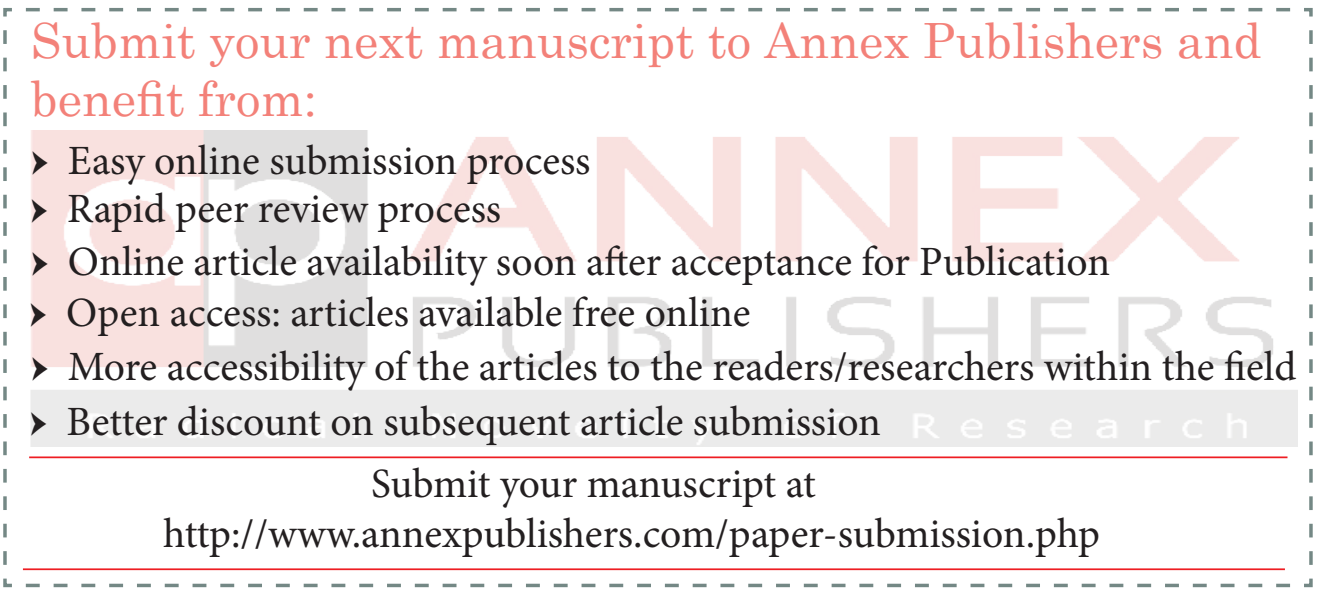

